significantly improved overall QOL, particularly with regard to 'interference with activities' and 'perceived sexual life' Urolife ${ }^{\circledR}$ subscores, compared with placebo. Notably, vardenafil did not improve postvoid residual urine volumes or peak urinary flow; however, baseline values for these parameters were near-normal, so whether vardenafil has clinically meaningful effects on bladder-outlet obstruction remains unclear.

The authors conclude that phosphodiesterase type 5 inhibitors could have a role in the treatment of LUTS secondary to BPH, particularly in men who also have erectile dysfunction.

Original article Stief CG et al. (2008) A randomised, placebo-controlled study to assess the efficacy of twicedaily vardenafil in the treatment of lower urinary tract symptoms seconday to benign prostatic hyperplasia. Eur Urol [doi:10.1016/j.eururo.2008.01.075]

\section{Prompt initiation of pharmacotherapy benefits patients with interstitial cystitis}

Interstitial cystitis (IC) is a painful, chronic bladder syndrome that is often diagnosed late-partly because the symptoms are shared with common conditions-and treated after considerable delay. Although pharmacologic treatment has proven ineffective for many patients with IC, pentosan polysulfate sodium (PPS) seems to be beneficial. In a post-hoc analysis of data from a randomized trial, Nickel et al. examined whether the interval between diagnosis of IC and initiation of PPS $(300 \mathrm{mg}$ daily for 32 weeks) affected the extent of symptomatic improvement.

The researchers identified patients with IC who had started PPS either $\leq 6$ months (early group; $n=57$ ) or $\geq 24$ months after diagnosis (late group; $n=46$ ). Only 62 participants completed the trial, but completion rates were similar between groups. At baseline and 32 weeks, the severity and extent of nuisance from symptoms were assessed by the O'Leary-Sant IC Symptom Index (ICSI) and IC Problem Index (ICPI), respectively.

At baseline, ICSI and ICPI scores were similar between the early and late treatment groups. At 32 weeks, however, improvement in both scores was significantly greater in the early group than the late group; the same trend was identified in subgroups of patients in whom treatment was initiated $\leq 3$ months or $\geq 36$ months after diagnosis.

Limitations of the study were that time since diagnosis rather than time since symptoms began - probably longer-was used and that $\sim 65 \%$ of participants had received at least one IC-related medication before the trial; nevertheless, the findings support previous reports of the benefit of early pharmacologic treatment for IC.

Original article Nickel JC et al. (2008) Time to initiation of pentosan polysulfate sodium treatment after interstitial cystitis diagnosis: effect on symptom improvement. Urology 71: $57-61$

\section{Personalized medicine for men with benign prostatic hyperplasia}

The $\alpha_{1}$-adrenoceptor (AR) antagonists tamsulosin hydrochloride and naftopidil, which are used to treat benign prostatic hyperplasia $(\mathrm{BPH})$, are subtype-selective; tamsulosin preferentially binds the $\alpha_{1 a}-A R$, whereas naftopidil preferentially binds the $\alpha_{1 d}$-AR. Men with BPH seem to have either predominant $\alpha_{1 a}$-AR or $\alpha_{1 d^{-}}$AR prostate-specific messenger RNA (mRNA) expression, and Kojima et al. have now examined whether this genetic difference explains the variation between patients' responses to treatment with subtype-selective $a_{1}$-AR antagonists.

The study included Japanese men with clinical BPH who were randomly allocated to receive tamsulosin $(n=33)$ or naftopidil $(n=28)$. Patients' levels of specific $\alpha_{1}$-AR mRNA expression were measured in total RNA extracted and amplified from prostate needle-biopsy samples obtained before randomization. Treatment efficacy was assessed by comparing baseline objective and subjective parameters of BPH with those after 12 weeks of treatment.

For the analysis, treatment groups were subdivided according to the predominance of

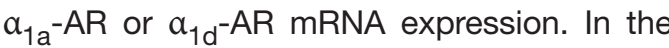
tamsulosin group, patients with $\alpha_{1 a}$-AR dominance $(n=22)$ responded better to the treatment than patients with $\alpha_{1 d}$-AR dominance. By contrast, naftopidil had increased efficacy in patients with $\alpha_{1 d^{-}}$AR dominance $(n=16)$ compared with those with $\alpha_{1 a}$-AR dominance $(n=12)$. 